MSO4-03

\section{The attachment of the lactobacillus surface-layer array to the bacterial cell}

Elisabeth Damisch ${ }^{1}$, Markus Eder ${ }^{1}$, Andela Dordic ${ }^{1}$, Krishna Mohan Padmanabha Das ${ }^{1}$, Ulla Hynönen ${ }^{2}$, Airi Palva ${ }^{2}$, Janet Vonck ${ }^{3}$, Monika Oberer $^{1}$, Tea Pavkov-Keller ${ }^{4}$

1. Institut of Molecular Biosciences, University of Graz, Graz, Austria

2. Faculty of Veterinary Medicine, University of Helsinki, Helsinki, Finland

3. Structural biology, Max-Planck-Institute of Biophysics, Frankfurt am Main, Germany

4. Institute of Molecular Biosciences, University of Graz and ACIB $\mathrm{GmbH}$, Graz, Austria

email: elisabeth.damisch@edu.uni-graz.at

Surface layers (S-layers) are 2D paracrystalline lattices of proteins or glycoproteins which cover the whole cell surface of many Archaea and Bacteria. Since these proteins are in close contact with their habitat they fulfil many vital tasks like bacterial adherence to other cells or substrates, protection against life-threatening conditions and maintenance of the cell shape [1]

S-layer proteins of lactobacilli species have a highly basic $\mathrm{pI}$ and are between $25-71 \mathrm{kDa}$ in size $[1,2]$. They are attached to the cell wall by interaction with lipoteichoic acids (LTA) [1]. It is reported that they are involved in auto- coaggregation and adherence and therefore are significant for the stimulation of gut dendritic cells by interacting with specific receptors [3]. Our goal is to characterize the surface layer proteins SlpA and SlpX of Lactobacillus acidophilus, which are both necessary to build up the protective S-layer coat. By changing the composition of the S-layer coat, the organism is able to adapt to the changing environmental conditions and threats, e.g. osmotic stress. For structure-function characterization, we designed several protein fragments. Soluble fragments were purified and subjected to crystallization. Optimized crystals of the C-terminal fragments, containing the LTA-binding domain, diffracted to 1.8 and 2.2 $\AA$. Crystal structures were solved by SeMet-SAD and the later by molecular replacement. To further characterize the binding of the S-layer to bacterial cell we performed NMR titration experiments and isothermal titration calorimetry measurements with the C-terminal fragment of the protein.

References:

[1] Pavkov-Keller, T. et al. (2011). Prog Mol Biol Transl, 103, 73-130.

[2] Hynonen, U. \& Palva, A. (2013). Appl Microbiol Biotechnol, 97, 5225-5243.

[3] Konstantinov, S. R. et al. (2008). Proc Natl Acad Sci U S A, 105, 19474-19479.

Keywords: S-layer, Proteins, Lactobacillus acidophilus
MSO4-04

\section{Synthesis and characterization of cross- linked lysozyme crystals filled with single- walled carbon nanotubes bionanomaterials}

Guillermo Escolano ${ }^{1}$, Rafael Contreras-Montoya ${ }^{2}$, Juan J. DíazMochón ${ }^{3}$, Luis Álvarez de Cienfuegos ${ }^{2}$, José A. Gavira ${ }^{4}$

1. Departamento de Química Orgánica, Facultad de Ciencias (UGR) and Laboratorio de estudios cristalográficos, Instituto Andaluz de Ciencias de la Tierra (CSIC-UGR), Granada, Spain

2. Departamento de Química Orgánica, Facultad de Ciencias (UGR), Granada, Spain

3. Departamento de Química Farmacéutica y Orgánica, Facultad de Farmacia, (UGR), Centre for Genomics and Oncological Research, Pfizer/UGR/Andalusian Regional Goverment, PTS Granada, Granada, Spain

4. Laboratorio de estudios cristalográficos, Instituto Andaluz de Ciencias de la Tierra (CSIC-UGR), Granada, Spain

email: gec@ugr.es

Novel bionanomaterials are hybrid materials that include the combination of biomolecules and inorganic substances to generate, enhance or support relevant properties. Bionanomaterials have useful applications in bio- and nanotechnology applications ${ }^{1,2}$.Among the biomolecules used to prepare hybrid materials, proteins have shown to be versatile materials thank to their capacity to self-assembly in crystalline form generating a porous network of nanometer size. The internal cavities of the protein have the ability to act as template ${ }^{3,4}$ and it gives the material the possibility to extrapolate nanoscale properties to macroscopic materials for practical applications. In this work, we present a new methodology to homogenously incorporate inorganic particles within protein crystals using dipeptide hydrogels as growth media. To exemplify this methodology, we have obtained lysozyme crystals incorporating single wallet carbon nanotubes at different concentration. Crystals were grown in Fmoc-PhePhe-OH hydrogels 5 . The influence of the nanotubes on the diffraction properties, hardness, enzymatic activity and conductivity will be presented and discussed, as well as a full characterization of these new materials.

References:

[1] Ruiz-Hitzky, E.; Darder, M.; Aranda, P.; Ariga, K. Advances in biomimetic and nanostructured biohybrid materials. Adv. Mater. 2010, 22, 323-336.

[2] Lu, Y.; Liu, J. W. Smart nanomaterials inspired by biology: Dynamic assembly of error-free nanomaterials in response to multiple chemical and biological stimuli. Acc. Chem. Res. 2007, 40, 315-323.

[3] M. Liang, L. Wang, R. Su, W. Qi, M. Wang, Y. Yua and Z. He. Synthesis of silver nanoparticles within cross-linked lysozyme crystals as recyclable catalysts for 4-nitrophenol reduction. Catal. Sci. Technol., 2013, 3, 1910-1914.

[4] O. L. Muskens, M. W. England, L. Danos, M. Li, and S. Mann Plasmonic Response of Ag- and Au-Infiltrated Cross-Linked Lysozyme Crystals. Adv. Funct. Mater. 2013, 23, 281-290.

[5] Conejero-Muriel, et al, Chem. Commun. 51, (2015), 3862 \& M. Conejero-Muriel, et al., CrystEngComm, 17, (2015), 8072.

Keywords: bionanomaterials, crystallization, carbon nanotubes 